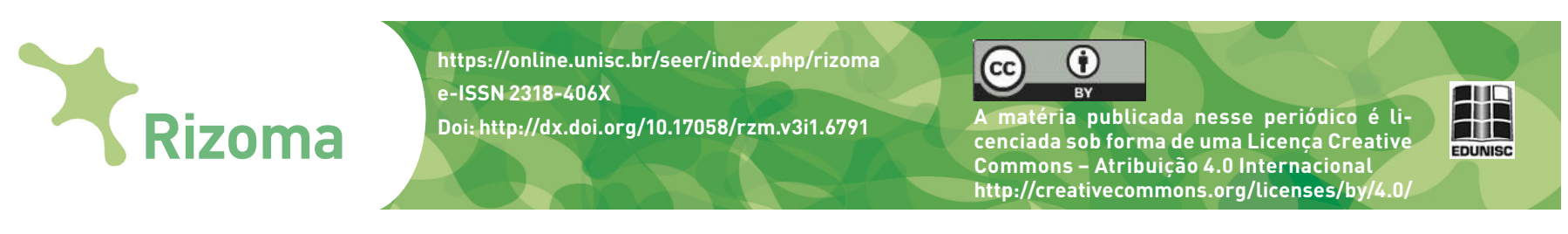

\title{
Propuesta de clasificación de blogs periodísticos
}

\section{Resumen}

Este trabajo presenta una propuesta de clasificación de blogs periodísticos, a partir de una investigación empírica comparativa entre bitácoras escritas por periodistas políticos brasileños y españoles. Para la realización de este estudio, se ha diseñado una metodología en la que se combina, además de la revisión bibliográfica, la observación sistemática, el análisis de contenido web y la entrevista en profundidad. El resultado de la investigación apunta sobre todo a tres tipos de blogs periodísticos encontrados con mayor frecuencia en las webs de periódicos de referencia: blogs de periodistas, blogs de columnistas y blogs de redacción.

Palabras clave: Ciberperiodismo; Blogs periodísticos; Brasil; España.

\section{Resumo}

Este trabalho apresenta uma proposta de classificação de blogs jornalísticos, a partir de uma pesquisa empírica comparativa entre blogs escritos por jornalistas políticos brasileiros e espanhóis. Para a realização deste estudo, desenhou-se uma metodologia que combina revisão bibliográfica, observação sistemática, análise de conteúdo web e entrevista em profundidade. O resultado da investigação aponta principalmente três tipos de blogs jornalísticos encontrados com maior frequência nos sites de jornais de referência: blogs de jornalistas, blogs de colunistas e blogs de redação. Palavras-chave: Ciberjornalismo; Blogs jornalísticos; Brasil; Espanha.

\begin{abstract}
This paper presents a proposal for the classification of journalistic blogs, from a comparative empirical research among blogs written by Brazilian and Spanish political journalists. For this study, it has been designed a methodology that combines, in addition to the bibliographical review, the systematic observation, analysis of web content and in-depth interview. The result of the research aims mainly three types of journalistic blogs most frequently found on the websites of reference newspapers: journalists blogs, columnists blogs and editorial blogs.
\end{abstract}

Keywords: Online journalism; Journalistic blogs; Brazil; Spain.
${ }^{1}$ Doctora en Periodismo por la Universidad Complutense de Madrid. Actualmente es profesora principal en el Programa de Periodismo y Opinión Pública de la Universidad del Rosario (Colombia) y profesora colaborada del Máster en Periodismo de la Universidad Estadual de Ponta Grossa (Brasil). 


\section{$Y_{\text {Rizoma }}$}

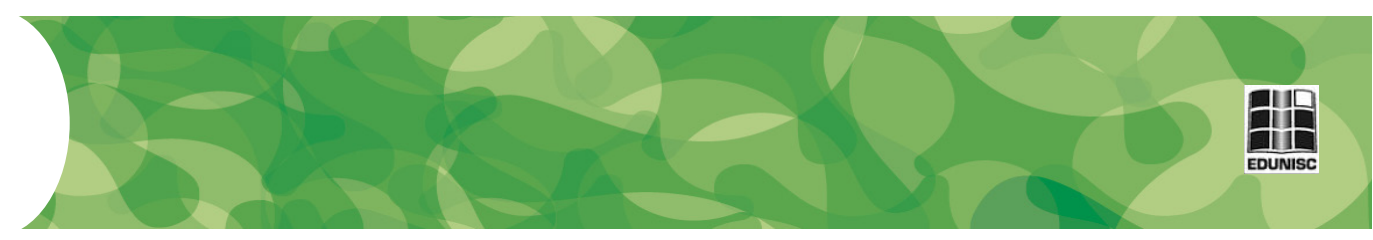

\section{Introducción}

Este trabajo presenta una propuesta de clasificación de blogs periodísticos o $j$-blogs ${ }^{2}$, resultado parcial de una investigación empírica más amplia que comprende el análisis de contenido web de diez blogs periodísticos políticos integrados en la web de periódicos de referencia de Brasil y España (COLUSSI RIBEIRO, 2013b).

En primer lugar abordamos cómo el concepto de blog periodístico aún es incipiente y está en desarrollo. La construcción del concepto parte de investigaciones que relacionan periodismo y blog, pero que en la mayoría de las veces no se han detenido en el estudio específico de bitácoras escritas por periodistas. De ahí, surge la importancia de debatir esa concepción y, como resultado de la presente investigación, proponer una definición de blog periodístico.

También exploramos las características propias de las bitácoras escritas por periodistas, en el sentido de diferenciarlas de los demás tipos de blogs. No hay duda de que parte de los elementos identificados en esos blogs están relacionados con la plataforma del cibermedio donde se alojan.

Por último hacemos hincapié en las taxonomías de los blogs periodísticos planteadas por investigadores de diferentes países (DOMINGO y HEINONEN, 2008; FOLETTO, 2009), a las que añadimos elementos identificados específicamente en nuestro análisis.

\section{Diseño metodológico}

Además de la revisión bibliográfica que ha servido de base teórica para fundamentar este trabajo, se ha seguido la recomendación de Díaz Noci y Palácios (2009) para combinar diferentes técnicas de investigación. En este caso, se ha utilizado la combinación de la observación sistemática abierta, del análisis de contenido web (HERRING, 2010) y de la entrevista en profundidad (DUARTE, 2005).

La observación sistemática abierta ha sido empleada para observar la generación de contenidos en los $10 j$-blogs políticos integrados en la web de periódicos de referencia de Brasil y España seleccionados para este estudio (véase cuadro 1), y también para delimitar las categorías de análisis de los blogs periodísticos.
${ }^{2}$ Abreviatura del término en inglés journalistic blog. 
Cuadro 1 - Blogs periodísticos políticos seleccionados para el análisis

\begin{tabular}{|c|c|c|c|}
\hline \multicolumn{2}{|c|}{ Brasil } & \multicolumn{2}{|l|}{ España } \\
\hline Blogs & Periódico & Blogs & Periódico \\
\hline 1. Josias de Souza & Folha de S.Paulo & $\begin{array}{l}\text { 1. ¡Qué paren las má- } \\
\text { quinas! }\end{array}$ & 20 Minutos \\
\hline 2. Presidente 40 & Folha de S.Paulo & 2. La sombra del poder & El Mundo \\
\hline 3. Blog do Noblat & O Globo & 3. Escolar.net & Público \\
\hline $\begin{array}{l}\text { 4. Diário de uma } \\
\text { repórter }\end{array}$ & O Globo & 4. La trinchera digital & Público \\
\hline $\begin{array}{l}\text { 5. José Roberto de } \\
\text { Toledo }\end{array}$ & $\begin{array}{l}\text { O Estado de } \\
\text { S.Paulo }\end{array}$ & & \\
\hline $\begin{array}{l}\text { 6. Joao Bosco Ra- } \\
\text { bello }\end{array}$ & $\begin{array}{l}\text { O Estado de } \\
\text { S.Paulo }\end{array}$ & & \\
\hline
\end{tabular}

Fuente: elaboración propia

El análisis de contenido web, una adaptación de la propuesta de Herring (2010), comprende diferentes categorías para el análisis de los elementos periodísticos de los j-blogs. Específicamente en este estudio, nos ha servido para verificar las características y perfil de los blogs periodísticos analizados. No hay dudas de que el análisis de contenido web y la observación sistemática, en este caso, han sido técnicas de investigación complementarias.

Con el objetivo de contrastar las informaciones y datos obtenidos en el análisis de contenido web de los $j$-blogs, se ha llevado a cabo la realización de entrevistas en profundidad semiaberta con cuatro de los periodistasblogueros: Ricardo Noblat, José Roberto de Toledo, Arsenio Escolar e Ignacio Escolar ${ }^{3}$.

Cabe aquí señalar los criterios adoptados para la selección de la muestra. Entre los criterios definidos, se encuentra a que el blog debe: 1) ser escrito por un periodista que se identifica en la bitácora y la actualiza por lo menos una vez por semana; 2) estar integrado a la web de un periódico de referencia de Brasil o España; 3) publicar posts acerca de la actualidad política; y 4) tener más de un año de actividad continua. Para seleccionar los diez blogs estudiados, se realizó una cartografía de los blogs en los ciberperiódicos de España y otra cartografía de los blogs en los ciberdiarios de referencia de Brasil.

La observación de los blogs periodísticos listados en ambas cartografías también ha contribuido para la construcción de la presente propuesta de clasificación de blogs periodísticos. En función de eso se realiza una taxonomía previa que, posteriormente, se verificó durante el análisis de contenido web de los diez j-blogs. En este caso, uno de los tipos de bitácoras periodísticas - blog de eventos - no ha sido identificado entre la muestra analizada, pero sí anteriormente.

Respecto a los límites temporales de la investigación que ha originado
${ }^{3}$ Las entrevistas fueron realizadas respectivamente en las siguientes fechas y lugares: el 30 de marzo de 2012 en Brasilia; el 18 de mayo de 2012 vía Skype; el 12 de julio de 2012 en Madrid; y el 10 de octubre de 2012 en Madrid. 
este artículo, se destacan cuatro momentos para el desarrollo de las diferentes etapas previstas: 1) La selección de la muestra tuvo lugar entre febrero y marzo de 2011;2) La observación sistemática de los j-blogs seleccionados se realizó en abril de 2011;3) El análisis de contenido web de los blogs se llevó a cabo entre enero y marzo de 2012; y 4) Las entrevistas en profundidad ocurrieron entre el 30 de marzo y el 10 de octubre de 2012.

\section{Del blog al blog periodístico}

La definición de lo que viene a ser un $j$-blog pasa por las siguientes fases: el crecimiento exponencial del número de blogs a partir del final de la década de 1990; la apropiación del formato para la publicación de relatos periodísticos, sobre todo, tras el atentado de 11-S y la Guerra de Iraq; y una última etapa, en la cual los cibermedios incorporan las bitácoras, adaptando su uso a la deontología periodística. De esa práctica se origina el blog periodístico.

Primeramente diferenciamos las bitácoras informativas de las periodísticas. Los blogs informativos suelen publicar informaciones especializadas sobre un sinfín de temas, tales como tecnología, redes sociales, cultura, etc. Esas bitácoras son mantenidas por distintos tipos de bloggers, que pueden ser desde un ciudadano corriente hasta un experto en el asunto o un periodista. En este sentido, Carrasco (2008) define blog informativo como aquel que amplía conocimientos en áreas específicas donde la mayor parte de los autores son expertos que tratan sobre los temas que más le interesan.

Son innúmeros los blogs informativos que se han convertido en un instrumento fantástico para los que deseaban hacer prensa con las propias manos. Hay numerosos de ejemplos, desde las bitácoras escritas por militares y ciudadanos que relataron la situación durante la Guerra de Iraq hasta blogs de moda, tecnologías y otros temas, que han conseguido profesionalizarse y atraer audiencia suficiente para ganar con publicidad.

Para las investigadoras brasileñas Quadros et al. (2005), la bitácora periodística forma parte de la categoría de los diarios informativos. "Los diarios informativos son de asuntos generales o sobre un tema específico, como cultura, economía, política y otros. Independiente del tema también pueden ser analíticos, de opinión, noticiosos o un mix de uno o más estilos" (QUADROS et al., 2005, p. 7).

Sin lugar a duda, el blog periodístico podría definirse como un diario informativo, tal y como proponen las mencionadas autoras, siempre y cuando se le añada que los responsables de actualizar este tipo de bitácora son periodistas, ya que suelen seguir los cánones de la profesión. En conformidad con Carrasco, consideramos que el blog periodístico también es "aquel que informa sobre hechos de interés público siguiendo las normas básicas de la redacción periodística al responder a las famosas W's de forma periódica y verosímil" (2007, p. 523).

En ese sentido, Araújo (2006) presenta una acepción de blog periodístico en la cual describe una definición general, considerando la 
responsabilidad del periodista-bloguero con la verdad. Para el autor, un blog es periodístico si presenta “un discurso basado en 'hacer-saber', expreso en el modo de verdad, y si está de acuerdo con las características de periodicidad, aparición pública, diversidad de contenido, interés general, actualidad y profesionalismo" (2006, p. 226).

Además, entendemos que a estas concepciones falta añadir que el $j$-blog es mantenido por un periodista, que trabaja para verificar e investigar la información, consultando fuentes informativas fiables antes de publicar el mensaje. Se supone que el periodista-bloguero realiza su labor sin olvidarse de una serie de obligaciones, normas y valores que forman parte de la deontología profesional (KOVACH y ROSENSTIEL, 2003).

Escobar (2009) y Foletto (2009) coinciden en situar el $j$-blog como una nueva categoría del ciberperiodismo, presentando la siguiente definición:

[... blogs periodísticos son aquellos cuyas direcciones son públicas y están asequible a cualquiera con acceso a Internet; que se dediquen en su totalidad o en la mayor parte del tiempo a divulgar acontecimientos reales dotados de actualidad, novedad, universalidad e interés; y cuyos blogueros se preocupen y se esfuercen para: publicar frecuentemente nuevos contenidos, aunque sin periodicidad fija o determinada; y divulgar sus blogs, convirtiéndoles en direcciones en la web ampliamente conocidas con el objetivo de atraer un número expresivo de internautas, es decir, una gran audiencia (que en Internet se expresa por el número de page views) (ESCOBAR, 2009, p. 225-226).

Para encuadrarse como una nueva categoría del ciberperiodismo, entendemos que ambos investigadores sitúan el blog periodístico dentro de la web de un cibermedio. No obstante, cuando el $j$-blog es independiente y posee dominio propio funciona como un cibermedio, que se rige de acuerdo con las normas de producción, distribución y de negocio establecidas por su autor. En los casos de bitácoras que forman parte de un cibermedio, la disposición de los elementos y la publicidad insertada no depende del periodista-bloguero, que sigue las pautas determinadas por la empresa periodística, como ocurre por ejemplo en el Blog do Noblat y Josias de Souza.

Defendemos, por tanto, que el blog periodístico es aquel mantenido por un periodista o un grupo de periodistas, integrado en la web de un cibermedio o con su propio dominio disponible para acceso público $\mathrm{y}$, además, sigue los cánones de la profesión periodística aprovechándose de las características de la blogosfera, tales como:

- Publicar contenidos que se refieren a acontecimientos reales mediante la adaptación de los valores noticia o criterios de noticiabilidad (WOLF, 1987; TRAQUINA, 2005) al formato blog. Entre los principales valores noticia se encuentran: la importancia e interés por los hechos, ${ }^{4}$ los acontecimientos novedosos y la actualidad, así como lo inesperado y el escándalo.
${ }^{4}$ Según Wolf (1987), el grado de importancia de la publicación de las noticias es el siguiente: si hay personajes famosos; el impacto sobre

la nación y el interés nacional; el número de personas implicadas; los hechos que tienen posibilidad de ser desarrollados. Respecto al nivel de interés, están la capacidad de entretenimiento, interés humano y de una composición equilibrada de noticias. 

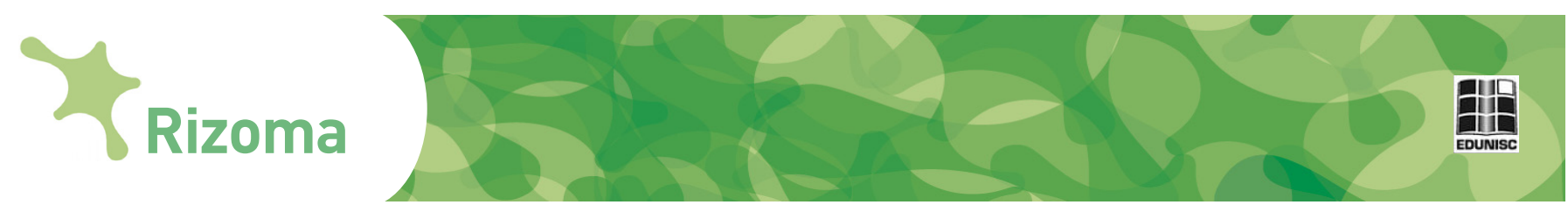

Tanto Wolf como Traquina destacan la categoría relativa a la competencia, en que los medios intentan conseguir las pautas antes de la concurrencia.

- Los periodistas-blogueros persiguen los valores y normas referentes a la deontología profesional, que incluyen el compromiso con la verdad, el trabajo de verificar la información antes de publicarla y el uso de objetividad en el tratamiento de los temas (KOVACH y ROSENSTIEL, 2003), buscando siempre la transparencia - característica común a los bloggers.

- El contenido publicado en las bitácoras periodísticas debe presentar un estilo y técnicas de redacción similares a las que se emplean en los demás medios de comunicación, considerando los géneros y la narrativa ciberperiodística (SALAVERRÍA, 2005; LARRONDO, 2008, PORTO y FLORES, 2012), sin olvidarse de explorar la hipertextualidad, multimedialidad e interactividad.

- La actualización del blog debe ser frecuente, aunque no haya una periodicidad fija.

- Debe permitir que los usuarios publiquen comentarios.

Además conviene aclarar que no todos los periodistas-blogueros mantienen bitácoras que se enmarcan en el universo de los blogs periodísticos. Por ejemplo, un periodista aficionado al ciclismo y a todo lo relacionado con el mundo de las bicicletas puede mantener un blog sobre este tema que no necesariamente sea un $j$-blog, ya que su interés en la publicación es más personal que profesional. Tal vez la cifra de este tipo de bitácoras escritas por periodistas sea más elevada que el número de blogs periodísticos.

Las bitácoras de grupos de investigación en el área de comunicación o de investigadores, a su vez, se encuadran entre los blogs académicos. En este abanico se incluyen, por ejemplo, Jornalismo \& Internet,${ }^{5}$ el blog del GJOL - grupo de investigación de periodismo en línea - de la Universidad Federal de Bahía en Brasil, y e-periodistas ${ }^{6}$ la bitácora mantenida por Ramón Salaverría, profesor de la Universidad de Navarra.

Tanto los blogs que ejercen el papel de observatorio de los medios de comunicación, como los escritos por expertos en medios sociales, no se clasifican como $j$-blogs. Como ejemplo, tenemos la bitácora del programa Observatório da Imprensa, ${ }^{7}$ de TV Brasil, que publica notas referentes al contenido del programa coordinado por el periodista Alberto Dines, y Periodistas $21,{ }^{8}$ de Juan Varela, que analiza los medios y el periodismo. La labor de estas bitácoras se asemeja a la de los watchblogs, ya que tratan de vigilar y analizar cómo actúan los medios de comunicación.
${ }^{5}$ Véase http://gjol.blogspot.com.br/.

${ }^{6}$ Véase http://e-periodistas. blogspot.com.br/.

${ }^{7}$ Véase http://www.obstv.blogspot. com.br/.

${ }^{8}$ Véase http://periodistas 21. blogspot.com.br/. 


\section{$Y_{\text {Rizoma }}$}

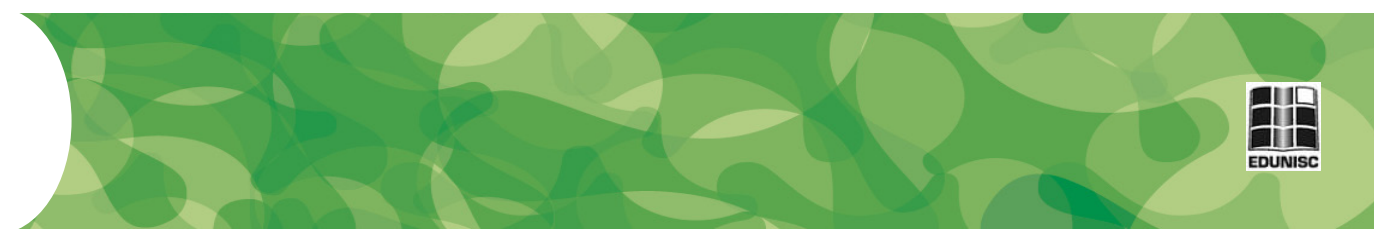

\section{Caracterización del blog periodístico como cibermedio}

Tras delimitar la concepción que tenemos de blog periodístico, en las siguientes líneas analizaremos las características del $j$-blog y su diferencial ante los demás tipos de bitácoras y cibermedios. Los blogs reciben diferentes clasificaciones entre las investigaciones sobre comunicación y periodismo, como un nuevo género (MATHESON, 2004; SINGER, 2005; LOWREY, 2006), un cibermedio (SALAVERRÍA et al., 2005) o una nueva categoría del ciberperiodismo (DOMINGO y HEINONEN, 2008; ESCOBAR, 2009; FOLETTO, 2009).

A diferencia de otros cibermedios, el blog periodístico viabiliza un periodismo más inmediato e interactivo (QUADROS et al., 2005), favoreciendo lo que Álvarez (2003) denomina instantaneidad de los medios digitales. Sin la obligación de seguir una guía de redacción y estilo o de tener el contenido chequeado por un editor (ROBINSON, 2006; NOBLAT, 2012), esas bitácoras son más ágiles a la hora de actualizarse.

Ese periodismo más inmediato incitado por los $j$-blogs exige la elaboración de contenidos a tiempo récord, en que no se piensa tanto en la estructura de la narrativa y en el estilo de redacción. Las publicaciones de un blog se asemejan a lo que Bradshaw (2007) nombra de borrador. Se trata de una primera impresión sobre un hecho, ya que la versión más completa y elaborada del relato periodístico se publicará en el medio de comunicación.

Al no ser regido por editores y no seguir un libro de estilo, los periodistas-blogueros tienen mayor libertad para crear contenidos. A pesar de reconocer la importancia de un libro de estilo para "homogenizar la información", Noblat (2012) subraya que una guía de redacción también limita la creatividad del periodista. En este sentido, el periodista-bloguero brasileño opina que la existencia de una guía favorece a los periodistas con menos experiencia profesional:

Tal vez para un periodista que esté empezando su carrera profesional la ausencia de un libro de estilo puede dejarlo desorientado. Pero este no es mi caso, que ya tengo mucho tiempo ejerciendo la profesión. En mi caso, el manual me ata más que sirve de orientación. (NOBLAT, 2012).

Además, como recuerda Singer (2005), el formato blog ofrece a los periodistas la posibilidad de ejercer la transparencia, sobre todo cuando se enlazan las fuentes informativas indicando al usuario el trayecto utilizado para obtener una información. Conviene recordar que esta práctica empezó en la blogosfera, pasando por los $j$-blogs, hasta llegar al ciberperiodismo.

A través de la transparencia los periodistas-blogueros demuestran que han verificado e investigado la información antes de publicarla. Es decir, posibilitan a los usuarios acceder a datos que confirman o amplían el mensaje original. De esa forma, están poniendo en práctica los 
elementos del periodismo: el compromiso con la verdad, la verificación de los hechos, la investigación objetiva y la transparencia (KOVACH y ROSENSTIEL, 2003).

El uso de enlaces en las bitácoras periodísticas favorece lo que Matheson (2004) titula periodismo de conexiones. En lugar de detenerse en la información propiamente dicha, los lectores de este tipo de blog exploran los diferentes caminos ofrecidos por la narrativa hipertextual. Este escenario ha colaborado para el desarrollo del periodismo contextualizado (PAVLIK, 2005), que actualmente es común en los cibermedios.

También forman parte del ciberperiodismo contextualizado documentos añadidos en formato de audio, vídeo, infografía digital, etc. Esa práctica que empezó en los blogs poco a poco ha sido incorporada por el periodismo y ha propiciado la creación de nuevas narrativas (COLUSSI RIBEIRO, 2012), como la hipermedia (EDO, 2003; SALAVERRÍA, 2005) y la transmedia (JENKINS, 2008), contribuyendo para el desarrollo de un periodismo transmedia (PORTO y FLORES, 2012).

La lógica de esas nuevas narrativas digitales favorece un periodismo más interactivo en el cual el usuario encuentra diversos caminos para experimentar la lectura no lineal. Incluso los $j$-blogs tradicionales tienden a publicar entradas no lineales e interactivas (COLUSSI RIBEIRO, 2013b). Así, también propicia un periodismo más participativo (QUADROS et al., 2005), que permite la colaboración del internauta en la generación de contenidos y ofrece espacio para que puedan escribir comentarios.

La conjunción de la hipertextualidad, multimedialidad e interactividad conllevan a la evolución de nuevos géneros en los j-blogs. Al utilizar dispositivos móviles para generar contenidos para sus bitácoras, los periodistas-blogueros crean nuevos géneros informativos. El Blog de José Roberto de Toledo publica posts vía Twitter a la vez que Escolar.net genera una entrada a través de un mensaje SMS. Ambos casos se refieren a notas informativas cortas móviles (COLUSSI RIBEIRO, 2013a), nuevos géneros informativos que en el futuro pueden incorporarse a los cibermedios.

Debido a algunos rasgos positivos atribuidos a los blogs, estos se han convertido en fuentes de información para otros medios: son implacables y rigurosos, no toleran informaciones y datos mentirosos publicados por periodistas y políticos; son una nueva arma contra la censura; son universales, globales; y son alternativos y cercanos, flexibles, directos y gratuitos. Asimismo, los $j$-blogs heredan esas características y sirven de fuente informativa para diferentes medios, como ocurrió con el Blog do Noblat sobre todo durante la cobertura del Mensalão ${ }^{9}$ (COLUSSI RIBEIRO, 2008).

Sin lugar a duda, el blog periodístico depende de diferentes tipos de autoridades periodísticas (MATHESON, 2004). Al contrario de un periódico, la bitácora no tiene su autoridad definida. Lo que ocurre es la renegociación de la autoridad periodística, ya que ese proceso es menos evidente en el blog. Dicho de otra forma, hay una malla de autoridad en la que el cibermedio avala el artículo y la calidad de la información, así como la escritura, que
${ }^{9}$ Se trata de un caso de corrupción del gobierno brasileño, que tuvo destaque en los medios en 2005. 
refuerzan la autoridad del blog. Así lo explica Matheson: "Una especie de malla de autoridad se construye en el weblog a través de la vinculación del contenido que le otorga un cierto estatus, mientras que el valor del artículo enlazado se refleja en la bitácora" (2004, p. 21).

A raíz de la autoridad de cada periodista construida gracias a su reputación profesional, hemos observado en los $j$-blogs analizados que, a excepción de Presidente 40, los demás blogs periodísticos de Brasil destacan el nombre del periodista-bloguero en lugar de acentuar el nombre del blog. Los dos más influyentes en el país, Blog do Noblat y Josias de Souza, han triunfado en internet debido a la autoridad periodística de ambos profesionales. Cabe subrayar que este fenómeno no se desarrolla de la misma forma en las bitácoras españolas analizadas, en las que se apuesta por el nombre del blog en primer plano.

Los tipos de blogs que presentan las características de la investigación tradicional periodística son aquellos editados por periodistas con experiencia en otros medios, que migraron a Internet y siguen manteniendo el rigor con el que producían los contenidos anteriormente en los medios tradicionales. Son blogs caracterizados principalmente como columnas que migraron a la web y se convirtieron en espacios más dinámicos e interactivos, con la posibilidad de actualizaciones continuas y de insertar comentarios de lectores.

Desde el punto de vista de la economía del trabajo, este movimiento de adaptación de los periodistas al formato blog consiste en una práctica y en un proceso a fin de proteger la ocupación (LOWREY, 2006). También muchos profesionales creen que la bitácora aumenta su trabajo, sobre todo, cuando se trata de blogs de redacción. Otros ven a los blogs como un antídoto contra la mercantilización que silencia las voces de medios de comunicación independientes (SINGER, 2005). También hemos identificado periodistas que viven profesionalmente del periodismo que practican en el blog, como es el caso de los periodistas que mantienen las siguientes bitácoras: $B \log d o$ Noblat, Blog de José Roberto de Toledo y Blog de Josias de Souza.

\section{Clasificación de los j-blogs}

En este apartado analizamos las principales taxonomías de blogs periodísticos y, a continuación, presentamos una propuesta de clasificación propia, que se basa en la investigación empírica y en el análisis de contenido web de los diez $j$-blogs políticos que forman parte de la muestra.

Una de las clasificaciones más conceptuadas es la propuesta de Domingo y Heinonen (2008, p. 6), en la cual diseñan una tipología de los llamados journalistic weblogs. Los autores dividen los blogs periodísticos en cuatro grupos:

- Blogs de ciudadanos: son aquellos escritos por la audiencia y que se ubican fuera de los cibermedios. Pueden adoptar diferentes roles, tales como comentarista de medios o watchblog, escritores especializados, periodistas aficionados. Los autores se refieren a las 


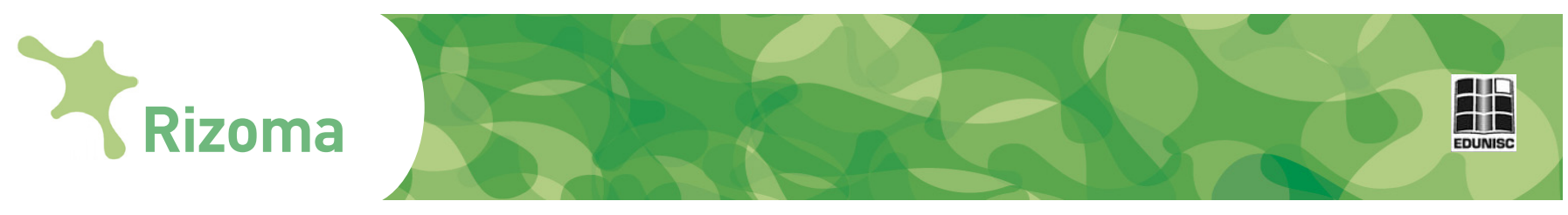

bitácoras de ciudadanos, las que tienen mayor representatividad en la blogosfera.

- Blogs de la audiencia: se tratan de bitácoras mantenidas por usuarios integradas a la web de los cibermedios. Son productos que forman parte de una serie de acciones para promover una relación de reciprocidad con la audiencia. La sección de blogs del público se convierte en un espacio en el cual se promueve un sentimiento de comunidad entre los lectores, fomenta el diálogo entre periodistas y usuarios, y mejora la lealtad de la marca. Por ejemplo, elpaís.com clasifica esas bitácoras como blogs de la comunidad.

- Blogs de periodistas: corresponden a bitácoras actualizadas por periodistas fuera del cibermedio. La principal característica de estos blogs es la independencia, que permite al periodista-bloguero una completa libertad editorial, sin tener que seguir los estándares delimitados por el medio de comunicación. Desde abril de 2012 Escolar.net dejó de formar parte de la web de Público.es y volvió a ser un j-blog independiente y, actualmente, está en la web de eldiario.es.

- Blogs de medios: son las bitácoras escritas por periodistas en los cibermedios. Este tipo de blog producido profesionalmente alcanza el máximo de desarrollo después de 2005. En el caso de blogs de redacción, a pesar de no tener requisitos editoriales estrictos como en las noticias, los editores supervisan los posts antes de la publicación. Además, esas bitácoras siguen muchas veces formatos y normas estándares establecidas por el cibermedio donde están alojadas.

Respecto a la clasificación de Domingo y Heinonen (2008) cabe el planteamiento de una excepción: algunos blogs escritos por periodistas que están alojados en un cibermedio puede que tengan la debida libertad para publicar y editar sus entradas bajo ninguna revisión anterior, aunque eso ocurra en casos aislados. A pesar de tener su blog hospedado en $O$ Globo, el periodista Noblat garantiza que tiene total libertad editorial y de publicación en su blog, ya que lo mantiene de forma independiente del periódico (COLUSSI RIBEIRO, 2013b).

No obstante, de acuerdo con la definición de blog periodístico y sus características, para la propuesta de clasificación de los j-blogs consideramos las dos últimas categorías indicadas por Domingo y Heinonen (2008), que denominamos de 1) j-blogs independientes y 2) j-blogs de medios.

El primer grupo se refiere a las bitácoras escritas por periodistas que no dependen de una institución. Es decir, son blogs independientes, con dominio propio y que siguen sus propios criterios para generar contenidos, gestionar la participación de usuarios e incluir o excluir algún elemento. Algunos de esos blogs comenzaron de forma independiente, pero más tarde fueron incorporados a webs de medios de comunicación, como es el caso del Blog do Noblat y Escolar.net.

Los $j$-blogs independientes suelen seguir los cánones de la actividad 
periodística aliados a la práctica del blogging. Entre ellos, se encuentran los blogs de periodistas sobre temas actuales y aquellos que funcionan como watchblogs u observatorio de prensa. Conviene recordar que tanto las bitácoras de expertos en comunicación como las de académicos no pertenecen a este grupo, ya que se tratan de blogs de expertos y de investigadores o profesores.

En cuanto al segundo grupo, que corresponde a los $j$-blogs integrados en la web de los cibermedios, la clasificación propuesta según la finalidad se divide en cuatro categorías:

- Blogs de periodistas: hemos denominado así considerando el grado de personalización periodística que este tipo de blog tiene. A pesar de formar parte de la sección de blogs del cibermedio, estas bitácoras son mantenidas por un periodista o un grupo de profesionales y no suelen recibir influencia editorial de los miembros de la redacción del medio de comunicación. Estos periodistas-blogueros generan sus contenidos o bien republican noticias o artículos de otros medios, de forma que el cibermedio no interfiere en su trabajo. Tanto es así que cada periodista-bloguero desarrolla su propio estilo de publicación. Corresponde a un grupo de periodistas con gran experiencia profesional en un área específica - política, cultura, deportes, etc. - que muchas veces tienen un contrato de prestación de servicio en el cual se comprometen con la actualización de sus bitácoras. De las bitácoras analizadas en esta investigación, hemos podido averiguar que los j-blogs brasileños Blog do Noblat, Blog de José Roberto de Toledo, Blog de Josias de Souza así como los españoles Escolarnet y iQue paren las máquinas! forman parte de este grupo.

- Blogs de columnistas: el objetivo de este tipo de bitácora es reproducir las columnas publicadas en la edición impresa del periódico. Las columnas de los periodistas se convierten en posts. Es decir, no se generan otros tipos de contenidos en los blogs de columnistas. No todos informan que la bitácora se dedica a republicar las columnas de la prensa, tampoco publican sus e-mails para que el público les pueda contactar. En este caso, la interacción entre periodistas-blogueros y el público es nula. Tanto La sombra del poder como La trinchera digital y Diário de uma repórter se incluyen en esta categoría.

- Blogs de redacción: son aquellos blogs mantenidos por una sección del ciberdiario - economía, salud, política, etc. - del cual un grupo de periodistas se encarga del proceso de generación de contenidos. A partir de los asuntos investigados para la publicación en la edición impresa o de algún material extra, los periodistas actualizan la bitácora. Lo más probable es que el contenido de este tipo de blogs reciba el visto bueno del editor antes de publicarse. En este caso, los periodistas-blogueros forman parte de la plantilla del medio de comunicación. Presidente 40, coordinado por Vera Magalhães, contaba con los redactores de la sección de política para actualizarse.

- Blogs de eventos: con la expansión del uso de Twitter como 
herramienta periodística, los cibermedios están utilizando el blog con menos frecuencia para la cobertura de eventos. No obstante, este tipo de bitácora da lugar a una especie de crónica en directo, estructurada en orden cronológico inverso. Esta práctica es común sobre todo en eventos deportivos. El portal $U O L$ de Brasil ha utilizado este modelo en la cobertura de los Juegos Olímpicos de Londres en julio de 2012. ${ }^{10}$ No obstante, en la muestra analizada en la presente investigación, no hemos identificado ningún blog que encaje en esta categoría.

Considerando el tipo de contenido publicado, conviene mencionar la clasificación de Foletto (2009). Conforme el autor, la bitácora periodística puede clasificarse en j-blog "columna", j-blog "portal” y j-blog "híbrido". El blog periodístico "portal" recibe este nombre gracias a la similitud de elementos que presenta en común a un portal de Internet. Este tipo de bitácora, según Foletto (2009), se caracteriza por actualizarse con contenidos de diferentes fuentes de información, como las agencias de noticias, medios de comunicación y colaboradores. Además, ofrece otros servicios no periodísticos a los usuarios, como una emisora de radio propia o un foro.

El blog periodístico "híbrido", a su vez, se constituye por el hibridismo de géneros y el uso potencial de la conversación, a través de la inserción de enlaces hipertextuales en los posts. "Este tipo de texto híbrido, similar al caracterizado por la columna, desarrolla su especificidad en la blogosfera a través del uso de enlaces para referenciar fuentes, citar ejemplos, reconstruir una discusión o reaprovechar un contenido publicado en otro sitio (FOLETTO, 2009, p. 84).

Aunque la propuesta de Foletto arroje luz sobre una clasificación que identifica los principales tipos de contenidos publicados en los j-blogs, presenta un contrapunto: debido al nivel de personalización que cada periodista-bloguero aplica a su bitácora, puede que no se encuadre en ninguna de estas tipologías por mezclar los tres tipos de contenidos. Los blogs de redacción, por ejemplo, pueden no encajar en estos modelos porque se dedican a publicar noticias de última hora de elaboración propia.

\section{Consideraciones finales}

Entre los tipos de bitácoras periodísticas presentados en la clasificación, destacamos los blogs de periodistas, blogs de columnistas y blogs de redacción. Los blogs de periodistas publican contenidos propios y recomiendan publicaciones de medios de comunicación, contribuyendo a la circulación de la información en el ciberespacio. De las bitácoras analizadas, forman parte de este tipo de blog: Blog do Noblat, Blog de Josias de Souza, Blog de José Roberto de Toledo, Blog de João Bosco Rabello, iQue paren las máquinas! y Escolar.net. Se destacan como bitácoras que ganaron protagonismo gracias a la autoridad de cada periodista, construida con base
${ }^{10}$ La cobertura está disponible en http://olimpiadas.uol.com.br/ao-vivo/2012/07/30/londres-agora.htm. 


\section{$\gamma_{\text {Rizoma }}$}

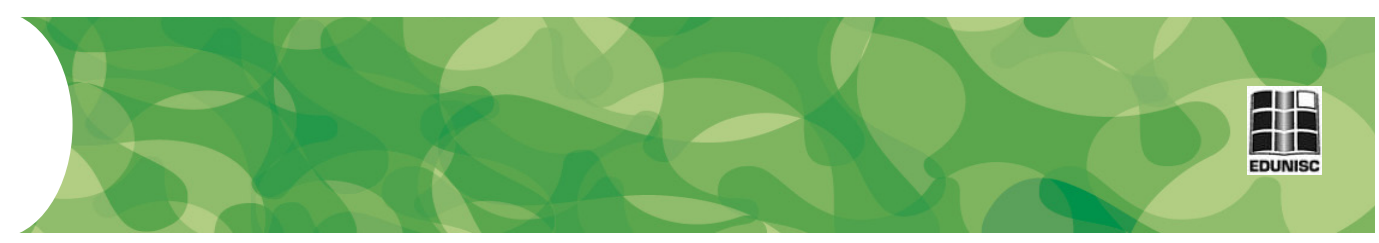

en su reputación profesional.

Los blogs de columnistas se caracterizan por reproducir en la bitácora columnas y artículos firmados por el periodista en la prensa escrita, como son el Diário de uma repórter, La sombra del poder y La trinchera digital. Es decir, el blog sirve tan solo como un espacio de reproducción de contenido, donde la interactividad es nula.

Por último, el blog de redacción es aquel mantenido por periodistas que trabajan en una sección de un periódico u otros medios de comunicación. En este caso, los profesionales utilizan la información bruta que consiguen con sus fuentes informativas a lo largo del día para actualizar la bitácora. En la muestra, el único blog que se encaja en este perfil es Presidente 40.

No menos importante son los blogs de eventos. No obstante, a raíz de la apropiación periodística de Twitter como herramienta para la cobertura de acontecimientos, sobre todo en el caso en los que se publica la información de forma instantánea, el periodista utiliza el blog con menos frecuencia para realizar la cobertura de un evento.

Conviene subrayar que la clasificación presentada en esta investigación no es una propuesta cerrada. Se refiere a una tipología que puede ser complementada por estudios que analicen otros tipos de blogs periodísticos $\mathrm{y}$, además, que examinen la generación de contenidos en diferentes países.

\section{Referencias}

ARAÚJO, Artur. Weblog e jornalismo: os casos de No Mínimo Weblog e Observatório da Imprensa (Bloi). Dissertação de mestrado. São Paulo: Universidade de São Paulo, 2006.

BRADSHAW, Paul. Amodel for the 21 st century newsroom: pt 1 - the news diamond, 2007. Disponible en: http://onlinejournalismblog.com/2007/09/17/amodel-for-the-21st-century-newsroom-pt1-the-news-diamond/. Acceso el out. 2010 .

CARRASCO POLAINO, Rafael. Fenómeno blog: información, comunicación, periodismo. In: FLORES VIVAR, J. et al. (ed.). Blogalaxia y periodismo en la red: estudios, análisis y reflexiones. Madrid: Editorial Fragua, 2008, p. 521-526.

COLUSSI RIBEIRO, Juliana. Blogs periodisticos y medios tradicionales: entre la competencia y la simbiosis. Un estudio de caso del Blog do Noblat. Madrid, España. Tesis de máster. Universidad Complutense de Madrid, 2008 .

La contribución de los blogs a la creación de un periodismo 

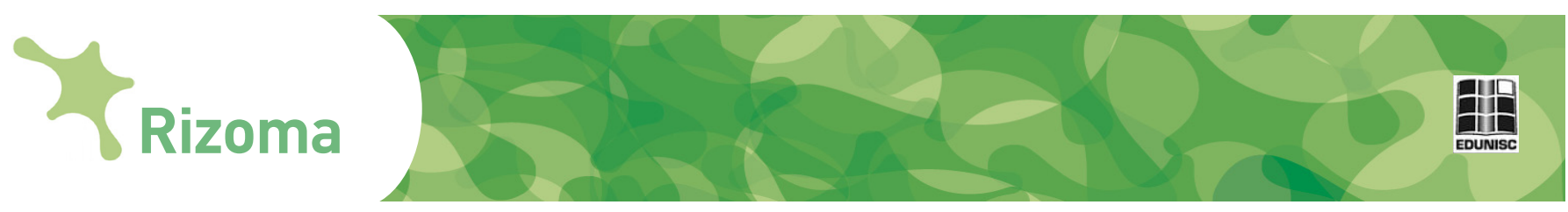

transmedia. In: PORTO RENÓ, D. et al. Narrativas transmedia. Entre teorías y prácticas. Bogotá, Editorial Universidad del Rosario, 2012, p. 229-252.

Nuevos géneros en el contenido de los blogs periodísticos publicado desde dispositivos móviles. In: João Canavilhas. (Org.). Notícias e mobilidade. Covilhã: Labcom - UBI, 2013a, p. 343-361.

. El blog periodístico como mini diario digital. Madrid: España. Tesis de doctorado. Universidad Complutense de Madrid, 2013b.

DÍAZ NOCI, J.; PALACIOS, M. (eds.). Ciberperiodismo: métodos de investigación. Una aproximación multidisciplinar en perspectiva comparada. Bilbao: Servicio Editorial de la Universidad del País Vasco, 2009.

DOMINGO, D.; HEINONEN, A. Weblogs and journalism. A typology to explore the blurring boundaries. Nordicom Review, n. 29, p. 3-15, 2008.

DUARTE, J. Entrevista em profundidade. In: DUARTE, J. (org.). Métodos e Técnicas de Pesquisa em Comunicação. São Paulo: Atlas, 2005, p. 62-83.

EDO, Concha. Periodismo informativo e interpretativo: el impacto de Internet en la noticia, las fuentes y los géneros. Sevilla: Comunicación Social Ediciones y Publicaciones, 2003.

HERRING, Susan. Web content analysis: Expanding the paradigm. In: HUNSINGER, J. et al. International Handbook of Internet Research. London: Springer Verlag, 2010, p. 233-249.

ESCOBAR, Juliana. Blogs como nova categoria de webjornalismo. In: AMARAL, A. et al. (orgs.). Blogs.com: estudos sobre blogs e Comunicação. São Paulo: Momento Editorial, 2009, p.217-235.

ESCOLAR, A. Entrevista [12 julio 2012]. Entrevistadora: Juliana Colussi Ribeiro. Madrid, 2012a.

ESCOLAR, I. Entrevista [10 octubre 2012]. Entrevistadora: Juliana Colussi Ribeiro. Madrid, 2012b.

FOLETTO, Leonardo Feltrin. O blog jornalístico: definição e características na blogosfera brasileira. Florianópolis, SC. Dissertação de mestrado. Santa Maria: Universidade Federal de Santa Catarina, 2009.

GARCÍA DE TORRES, Elvira. Estudio internacional de las herramientas de participación en la prensa digital. In: (coord.) Cartogra- 


\section{$\gamma_{\text {Rizoma }}$}

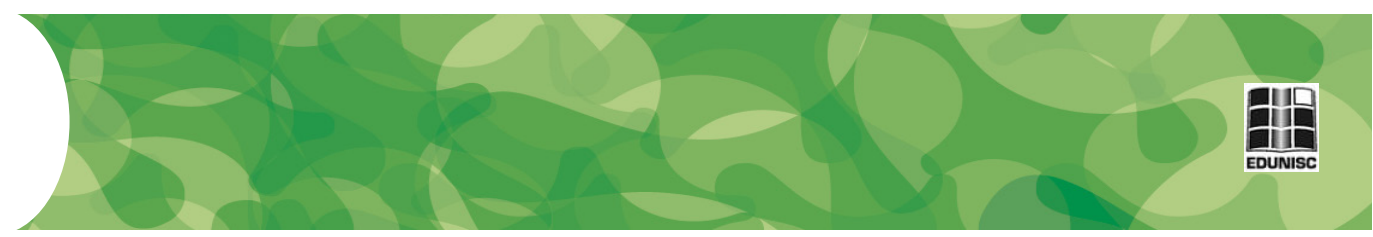

fía del periodismo participativo. Valencia: Tirant Humanidades, 2012, p. 59-120.

JENKINS, Henry. Convergence culture: la cultura de la convergencia de los medios de comunicación. Barcelona: Paidós, 2008.

KOVACH, B.; ROSENSTIEL, T. Los elementos del periodismo. Madrid: El País, 2003.

LASICA, J. Blogs and journalism need each other. Nieman Reports, p.70-75, 2003. Disponible en: http://www2.gvsu.edu/pearm/wrt380/p1/Content $\% 20$ Development\%20Resource2.pdf. Acceso el mai. 2010.

LARRONDO URETA, Ainara. Los géneros en la redacción ciberperiodistica. Contexto, teoría y práctica actual. Bilbao: Servicio Editorial de la Universidad del País Vasco, 2008.

LOWREY, Wilson. Mapping the journalism-blogging relationship. Journalism, London, 7 (4), p. 477-500, 2006.

MESO, K.; B. PALOMO. Los blogs de autor en los ciberdiarios de referencia españoles. Prisma.com, n. 7. 2008. Disponible en: http://revistas.ua.pt/ index.php/prismacom/article/viewFile/675/pdf. Acceso el set. 2012.

MESO, K. et al. Ferramenta para análise de blogs em cibermeios. In: PALÁCIOS, M. (org.). Ferramentas para análise de qualidade no ciberjornalismo. Vol. 1, Modelos. Covilhã, Labcom , 2011, p. 51-80.

NOBLAT, R. Entrevista [30 mar 2012]. Entrevistadora: Juliana Colussi Ribeiro. Brasília, 2012.

PAVLIK, John. El periodismo y los nuevos medios de comunicación. Barcelona: Paidós, 2005.

SALAVERRÍA, Ramon. Redacción periodistica en Internet. Navarra: Eunsa, 2005 .

PORTO RENÓ, D.; FLORES VIVAR, J. Periodismo transmedia. Madrid: Fragua, 2012.

QUADROS, C. et al. Blogs e as transformações do jornalismo. Revista da Associação dos Programas de Pós-graduação em Comunicação (e-Compós), p. 1-21, 2005. Disponible en: http://www.compos.org.br/seer/index. php/e-compos/article/viewFile/38/38. Acceso el jul. 2012.

ROBINSON, Susan. The mission of the j-blog. Recapturing journalistic au- 


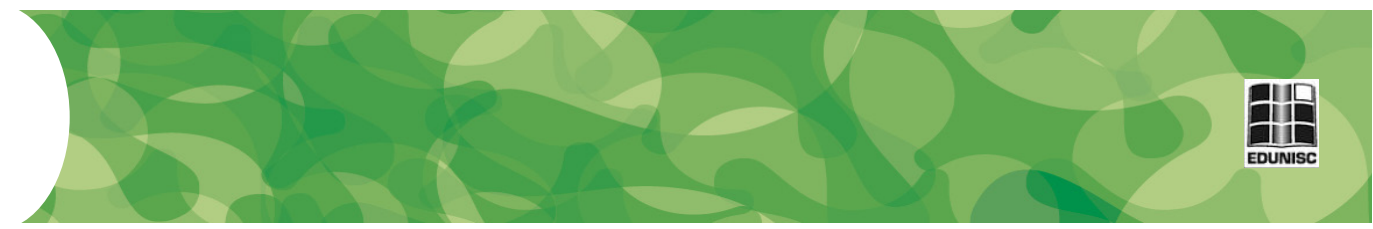

thority online. Journalism, 7 (1), p. 65-83, 2006.

SINGER, Jane. The political j-blogger. 'Normalizing' a new media form to fit old norms and practices. Journalism, 6 (2), 2005, p. 173-198.

TOLEDO, J. R. Entrevista. Entrevistadora: Juliana Colussi Ribeiro. Vía Skype, 2012. Acceso el mai. 2012.

TRAQUINA, Nelson. Teorias do jornalismo. A tribo jornalística uma comunidade interpretativa transnacional. Florianópolis: Insular, 2005.

WOLF, Mauro. Teorias da comunicação. Lisboa: Editorial Proença, 1987. 